\title{
THE BOOKSHELF OF THE UNIVERSITY LIBRARY IN PULA IS (NO LONGER) THE LAST STATION OF THE TRAVEL
}

\author{
Damjana Frančić
}

\begin{abstract}
When arriving by train from the North to Istria, the last stop is the railway station in Pula, so the Library is the last station for travel books. We searched our fund for the travelogues exhibition and found that we have very little travelogues about Istria, and disproportionately more travelogues about distant parts of the world. The catalogs of other libraries in Europe and the world show many travel books about Istria, which are digitalised and now can be read on the internet.
\end{abstract}

Keywords: Digitalised Travelogues, Istria, University Library in Pula

Travelogues are the oldest form of tourist promotion. For today's visitors to the Adriatic coast at the time of mass tourism it is hard to believe that our coast has been until recently scarcely known in Europe. At the beginning of the 20th century travelers reported that Dalmatia was less known than Africa, that this was "the Middle East", "wild Europe", "the edge of the Orient", a periphery.

Travel has always meant education. In the Middle Ages, pilgrims looked at the travels to the sacred places as spiritual discipline, the 
acquisition of divine knowledge, and the enterprise that will bring them a reward in the after life. Sudden development of foreign trade in the 16th century after geographic discoveries influenced publication of practical guidances on travel and manuals for merchants, starting from the age of when it was time for travel (between ages 40 and 56). In the lists of products to be searched for in the distant countries there are left many informal, unpublished notes about paying attention to the customs of the countries to which they were traveling. In these manuals it is rarely recommended for travelers to describe natural beauties, they have to look at how the country is populated, whether the land is fertile and how it yields. Romanticism later brought enjoyment to the wild landscapes and nature, and in the 18th century a new interest in archeology is represented by travelogues such as Alason's, which was dedicated to the display of ancient monuments.

Travelers had unclear misconceptions about the delimitation of ethnic groups, and had little knowledge of the boundaries and names of geographical or administrative areas. These old works have a disadvantage for us, they have no data about the things we are interested today; they list new and old names of places, talk about administrative institutions, but don't describe homes or everyday life. The travel writers were asked to provide information about the geographic location and the origin of the place names in the manuals and from these manuals they often accepted attitudes about the characteristics of the people in the countries they have traveled to. This can be seen in description of the inhabitants of our regions. Travelers didn't come into contact with the inhabitants, although they described the characteristics of these people. The inhabitants avoided foreigners especially if they were traveling with Turkish military escorts. So, travelers didn't have the opportunity to observe the lives, customs, looks and costumes of the hosts.

The journey was a difficult venture that needed enough strength and endurance. Traveling to Western Europe was relatively safe with the help of a chariot. Temptations for passengers were a bad road situation, poor supply, shortcomings that were exposed at a time when a stranger was a rarity and provoked hostility or at least anxiety and a conspiquous physical effort to travel. With the transition to a province under the rule of the Turks, difficulties were increasing. Trips were more expensive than 
today because of the real cost of transportation, accommodation and nutrition, but also because they lasted longer and could have come to an unexpected retention. It is difficult to estimate how much did the trip cost, but probably ten times more than today.

Due to its geographic position, the Croatian coast was the preferred natural bridge of the European West and the Middle East. In the 15th century there was a rise of pilgrimage on the Adriatic. Improving economic opportunities in northern Europe enabled noblemen, churchmen and not particularly rich knights to embark on daring and long-lasting tourist adventures. The journey usually lasted six months or longer, depending on the route they were passing. The Turks were robbing the Balkans, and as the Middle East was stabilizing, the northerners rushed to see the southern countries. It was easier for the Germans, the French, the Dutch and the Danes to cross the Alps and come to Venice to use ships than to go on an unsafe journey through the Biscay Bay and Gibraltar to get to Palestine. The Venetians have established a tourist service and advertised shipping services and they traveled as guides. Caravans were exhausted and hungry when they arrived to Venice, where cunning hosts would hold them down for weeks because of weak winds, storms and pirate ambitions, forcing travelers to spend their money. The pilgrims themselves had to buy daily travel necessities, like spoons and plates for themselves. They bought mattresses, blankets, domestic animals for food on the way, food for those animals, wine and medicines, necessary for long sailing trip, but pirates and thunder storms were waiting for them on the sea. The passengers packed into steerage of the ship were exposed to vermin, dirt, sicknesses, days without fresh food and water. Contracts of the Venetian carriers and the pilgrims, due to such conditions, had a clause regulating the procedure with the body and property of passengers who would die on the trip. Before starting the journey the tip was to supply the traveler with wine he usually drank, because good wine could only be bought upon the arrival to Zadar and later to Dubrovnik.

The islands and shores of the Adriatic coast provided a shelter for sailors and ships from storms that could take human lives. Our ports were the only place where they came into contact with inhabitants of deeper inland territories. Those who sailed along the Adriatic coast had a lively 
imagination when they wrote about where the territory named Croatia, Dalmatia, Bosnia started and where the unclearly restricted territory that they called Sklavonia ended.

Travel books that mostly refer to other countries - Syria and the Middle East, Equatorial Africa, India and Asia, Australia, Central and North America, the North Pole and of course the Old Europe - have reached the last station on their journey (and arrived into our Library) while the travelogues that describe Istria are stored in other places in Europe. Today we have the opportunity to inspect the texts that describe our regions, because a lot of the books have already been digitalised and are available in this way, so we have to change the paradigm of our time Europe has first met us before almost some 500 years ago and now we can read about it.

There already exists a project of the Institute of Art History from Zagreb: "Dalmatia - a destination of European Grand Tour in the 18th and 19th century", on the website http://grandtourdalmatia.org. There is also a similar project about the Italian part of the Adriatic coast the data is collected by the project http://www.viaggioadriatico.it/ of International Interuniversity Center of Studies on the Adriatic Journey, University of Salento, from Lecce, Italy. We should also mention The Archive of Adriatic Studies in Venice asa.archiviostudiadriatici.it, and a project pro.europeana. eu/project/europeanatravel.

Below is a selection of more than hundred and seventy of such books which describe Istria during all those years, with links to the full texts visible on the internet:

\section{Travelogues and Field Guides to Istria}

1462. - Wey, William: The Itineraries of William Wey, fellow of Eton college, To Jerusalem, A.D. 1458 and A.D. 1462 ..., London, J.B. Nichols and sons /from manuscript/, 1857. https://archive.org/details/ cu31924013279462/page/n01

1506. - Ellis, Henry: The pylgrymage of Sir Richard Guylforde to the Holy Land, A.D. 1506., London, Camden Society, 1851. https://archive. org/details/pylgrymageofsirr00ellirich/page/n01 
1551. - Alberti Leandro F.: Descrittione di tutta Italia, nella quale si contiene il sito di essa, l'origine, et le signorie delle città, \& delle castella, co i nomi antichi, \& moderni, i costumi de popoli, le conditioni de Paesi ..., in Vinegia, app. P. de e N. da Sabbio https://babel.hathitrust.org/cgi/ pt?id=ucm.5316865467; view=1up;seq=1

1553. - Giustiniano, Giovanni Battista: Itinerario di - Breve descrizione d'Istria, Monumenta spectantia historiam slavorum meridionalium, Vol. VIII. Comm. et Relat. Venetae, Tomus II. Ed. Acad. Scient. et Art. Slavorum Merid., Rx Off. Soc. Typ., in Tab. Libr. L. Hartman (Zagrabiae, 1877) http://www.istrianet.org/istria/literature/periodicals/ monumenta/1877_Monumenta.pdf

1573. - Pinargenti, Simon: Isole che son da Venetia nella Dalmatia, et per tutto l'Arcipelago, sino à Costantinopoli, con le terre più notabili di Dalmatia, nuouamente poste in disegno a beneficio de gli studio si di geografia, Vinegia https:/exhibits.stanford.edu/renaissance-exploration/ catalog/hw418vr3055

1574. - Camocio, Giovanni Francesco: Isole famose, porti, fortezze, e terre maritime sottoposte alla Ser.ma Signoria di Venetia, ad altri Principi Christiani, et al Sig.or Turco..., Venezia, Libraria del segno di S. Marco https://exhibits.stanford.edu/renaissance-exploration/catalog/ cb466fd5827

1584. - Cà da Mosto, Alvise - Rampazetto, Francesco: Il Portolano Del Mare: Nel Qval Si Dichiara Minvtamente Del Sito Di Tutti i Porti; Qvali Sono $\mathrm{Da}$ Venetia In Leuante, $\&$ in Ponente ..., in Venetia, appresso gli Heredi di F. Rampazetto http://digital.onb.ac.at/OnbViewer/viewer. faces?doc=ABO_\%2BZ260077302

1598. - Rosaccio, Gioseppe: Viaggio da Venetia a Costantinopoli per mare, e per terra, \& insieme quello di Terra Santa ... Nel quale, oltre a settantadui disegni, di geografia, e corografia si discorre, quanto in esso viaggio..., Venezia, G. Franco https://books.google.hr/ books?id=x7DkwbREMl8C\&dq=istria+viaggio $\&$ hl $=$ hr\&source $=$ gbs navlinks_s 1600. - Serlio, Sebastiono: Tutte l'opere d'architettura et prrospetiva 
... dove si mettono in disegno tutte le maniere di edificij, e di trattano di quelle cose, che sono più necessarie a sapere gli architetti ..., Vinegia, Francesco de' Franceschi https://www.e-rara.ch/download/ pdf/108882? name=Tutte $\% 201 \% 27$ opere $\% 20 \mathrm{~d} \% 27$ architettura $\% 20$ et $\% 20$ prrospetiva\%20ie\%20prospettiva\%20di\%20Sebastiano\%20Serlio 1608. - Mundy, Peter: The Travels of Peter Mundy in Europe and Asia, 1608-1667., Vol. I. Travels in Europe, 1608-1628., Cambridge, Hakluyt Society, 1907. https://archive.org/details/travelspetermun00mundgoog/ page/n2

1609. - Lithgow, William: The Totall Discourse of the rare Adventures and poinefull Peregrinations of long nineteene years Travailes from Scotland to the most famous Kingdomes in Europe, Asia and Affrica, Glasgow, J. MacLehose, 1906. https://archive.org/details/ totalldiscourseo00lithuoft/page/n1

1611. - Manzuoli, Nicolò: Nova descrittione della Provincia dell'Istria, con la vita delli santi, et sante di detta prouincia raccolte dalle legende loro antiche, [et] autentiche conseruate nelli archivi delle chiese ..., in Venetia, appresso G. Bizzardo http://digital.onb.ac.at/OnbViewer/viewer. faces?doc=ABO_\%2BZ178211605

1612. - Megiser, Hieronymus - Christalnick, Michael Gothard: Annales Carinthiae, Das ist Chronica Des Löblichen Ertzhertzogthumbs Kharndten: Darinn ..., Leipzig, Lamberg http://reader.digitalesammlungen.de/de/fs1/object/display/bsb10934609_00001.html 1615. - Sandys, George: A Relation of a Journey Begun an Dom. 1610. Foure Bookes Coutaining a Description of the Turkish Empire, of Aegypt, of the Holy Land, of the Remote Parts of Italt, and Islands Adjoyning, London, Barren https://books.google.hr/books?id=6jxFnu9A_ MYC\&printsec $=$ frontcover\&hl $=\mathrm{hr} \# \mathrm{v}=$ onepage\& $\mathrm{q} \& \mathrm{f}=\mathrm{false}$ 1620. - Porcacchi, Tommaso, Porro, Girolamo: L' isole più famose del mondo descritte da Thomaso Porcacchi da Castiglione arretino e intagliate da Girolamo Porro padovano con nova aggionta..., Padova, Paolo et Francesco Galignani fr. https://books.google.hr/ books?id=gbeH71iBinkC\&pg=PP7\&lpg=PP7\&d 
1621. - Sandys, George: Relation of a Iourney begun AD. 1610. Foure Bookes Containing a Description of the Turkish Empire, of Aegypt, of the Holy Land, of the Remote parts of Italy, and Islands adioyning, London, W. Barrett https://archive.org/details/relationofjourne00sand/page/n3 1629. - Rithi, Biagio: Commentari della guerra moderna passata nel Friuli, e ne confini dell'Istria, e di Dalmatia, Divisi in 8 libri, Trieste, Turrini http://reader.digitale-sammlungen.de/de/fs1/object/display/ bsb10000394_00003.html

1636. - Blount, Henry: A voyage into the Levant, a Breife relation of a iourney lately performed by Master H. B. Gentleman, from England by the way of Venice, into Dalmatia, Sclavonia, Bosnah ..., London, by I. L. for Andrew Crooke https://babel.hathitrust.org/cgi/pt?id=miun. alt2311.0001.001; view=1up;seq=1

1652. - François, Jean P.: La science de la géographie divisée en trois parties ... Première partie, des divisions géographiques, Hardy (Rennes) https://gallica.bnf.fr/ark:/12148/bpt6k58183548.r=Istrie?rk=6309044;0 1656. - Duval, Pierre: Le voyage et la description d'Italie montrant exactement les raretez et choses remarquables qui se trouvent es provinces..., Paris, G. Glouzier https:/gallica.bnf.fr/ark:/12148/ bpt6k83845h.r=Istrie?rk=150215;2

1663. - Payen: Les voyages de Monsieur Payen, où sont contenues les descriptions d'Angleterre, de Flandre, de Brabant, d'Holande, de Dennemarc, de Suède, de Pologne, d'Allemagne et d'Italie..., Paris, E. Loyson https://gallica.bnf.fr/ark:/12148/bpt6k5684438q. $\mathrm{r}=$ Istrie? $\mathrm{rk}=2789713 ; 2$

1678. - Spon, Jacob: Voyage d'Italie, de Dalmatie, de Grèce et du Levant, fait aux années 1675 et 1676, par Iacob Spon, Docteur Medecin Aggregé à Lyon, et George Vvheler, Gentilhomme Anglois, vol. I., Lyon, Chez Antoine Cellier le fils https:/gallica.bnf.fr/ark:/12148/bpt6k85322z/ f1.image 1699. - Dumont, Jean: Voyages de Mr Du Mont en France, en Italie, en Allemagne, à Malthe et en Turquie. Contenant les recherches 
\& observations curieuses qu'il a faites en tous ces païs..., É.

Foulque (La Haye) https://gallica.bnf.fr/ark:/12148/bpt6k56797366.

$\mathrm{r}=$ Istrie? $\mathrm{rk}=3047225 ; 2$

1700. - La Sacrosanta Casa Di Nazaret: Per disposizione Diuina di Galilea da gl'Angeli trapassando la Siria, Macedonia, Albania, e Dalmazia, miglia Italiane 1895. su trasportata à Tersatto nell'Istria, e di là per l'Adriatico miglia 145, à Loreto, Macerata, Er. del Pann http://reader.digitalesammlungen.de/de/fs1/object/display/bsb11096301_00005.html 1700. - Naldini, Paolo: Corografia ecclesiastica o' sia descrittione della città, e della diocesi di Guistinopoli, detto volgarmente Capo d'Istria, Venezia, Gierolamo Albrizzi http://reader.digitale-sammlungen.de/de/fs1/ object/display/bsb10005206_00001.html

1707. - Lecoq, Renaud: Le Parfait geographe, ou L'art d'apprendre aisément la geographie et l'histoire, par demandes et par réponses. Tome 1. Revûe, corrigée \& augmentée des moeurs, de la religion, \& du gouvernement de chaque nation, enrichie de cartes geographiques ..., Paris, I. Debats https://gallica.bnf.fr/ark:/12148/bpt6k97830724. $\mathrm{r}=$ Istrie? $\mathrm{rk}=21459 ; 2$

1716. - Bunou, Jacques-Philippe: Abregé de geographie ou Methode pour apprendre facilement la disposition des diverses parties du globe terrestre ... Avec un Dictionnaire geographique tres-exact.., A Rouen chez Richard \& Nicolas Lallemant https://gallica.bnf.fr/ark:/12148/bpt6k9787479n. $\mathrm{r}=$ Istrie? $\mathrm{rk}=3025766 ; 0$

1716. - Lenglet Du Fresnoy, Nicolas: Methode pour etudier la géographie, dans laquelle on donne une description exacte de l'Univers, \& formée sur les observations de messieurs de l'Academie royale des sciences. Tome 1, Paris, C.-E. Hochereau https://gallica.bnf.fr/ark:/12148/bpt6k97864140. $\mathrm{r}=$ Istrie? $\mathrm{rk}=21459 ; 2$

1727. - Briemle, V.: Die durch die drey Theile der Welt, Europa, Asia und Africa, besonders in denselben nach Loreto, Rom, Monte-Cassino, nicht minder Jerusalem, Bethlehem, Nazareth, Berg Sinai, [et]c..., München, Ver. G. Christoph Weber https://babel.hathitrust.org/cgi/ pt?id=nyp.33433000402721;view=1up;seq=1 
1734. - Travels of Three English Gentlemen, from Venice to Hamburgh, in The Harleian Mischellany, a Collection of scarce, curious, and entertaining phamphlets and tracts, Vol. XI. Printed for Robert Dutton (London, 1810) https://books.google.hr/books/about/The_Harleian_ Miscellany.html?id=Qh0wAAAAYAAJ\&redir_esc $=y$

1735. - Fäsch, J. Rudolph: Kriegs-, Ingenieur-, Artillerie- und Seelexicon: worinnen alles was einem Officier, Ingenieur, Artilleristen, und Seefahrenden ..., Dresden, F. Hekel https://babel.hathitrust.org/cgi/ pt?id=nyp.33433008512638; view=1up;seq=1

1743. - Pococke, Richard: A description of the East, and some other countries, vol. 1-2, London, W. Bowyer, 1743-1745. https://babel. hathitrust.org/cgi/pt?id=gri.ark:/13960/t06x1h58n;view=1up;seq=1 https://babel.hathitrust.org/cgi/pt?id=gri.ark:/13960/ t8vb09c39; view=1up;seq=1

1744. - D’Anville, Jean-Baptiste Bourguignon: Analyse géographique de l'Italie, dédiée à monseigneur le duc d'Orléans, premier prince du sang, Paris, Vve Estienne https://gallica.bnf.fr/ark:/12148/bpt6k5652605k. $\mathrm{r}=$ Istrie? $\mathrm{rk}=6394881 ; 2$

1747. - Scotto, Francesco: Itinerario d'Italia in questa nuova edizione abbellito di rami, accresciuto, ordinato ed emendato, Ove si descrivono tutte le principali citta d'Italia ..., Roma, Fausto Amidei Mercante di Libri al Corso https://babel.hathitrust.org/cgi/ pt?id=ucm.532021098x;view=1up;seq=1

1750. - Donati, Vitaliano - Lionardo, Sesler: Della Storia naturale marina dell' Adriatico, giuntavi una lettera di Lionardo Sesler, Venezia , F. Stoati http://reader.digitale-sammlungen.de/de/fs1/object/display/ bsb10051082_00005.html

1752. - De Lacroix, Louis-Antoine Nicolle: Géographie moderne, Tome 1, précédée d'un petit traité de la sphere \& du globe; ornée de traits d'histoire naturelle \& politique \& terminée par une géographie ecclésiastique..., Paris, J.-T. Herissant https://gallica.bnf.fr/ark:/12148/ bpt6k9788773h.r=Istrie?rk=21459;2 
1753. - Salmon, Thomas: Continuazione dell'Italia o sia descrizione degli altri stati veneti, del Dogado, Trivigiano, Friuli, Istria, Dalmazia e Levante Veneto, Venezia, presso Giambatista Albrizzi Q. Gir. https:// books.google.hr/books?id=Qc5hAAAAcAAJ

1756. - Clerget, M.: Géographie pour les jeunes gens dans un goût nouveau. Ou Abrégés de l'arithmétique, de la sphére, et de la géographie, Paris, Babuty fils https://gallica.bnf.fr/ark:/12148/bpt6k97860455. $\mathrm{r}=$ Istrie? $\mathrm{rk}=6094450 ; 4$

1759. - Bruzen de La Martinière, Antoine-Augustin: Abrégé portatif du dictionnaire géographique de La Martiniere, Tome 2, Paris, Le Mercier https:/gallica.bnf.fr/ark:/12148/bpt6k97851367.r=Istrie?rk=21459;2

1768. - Lenglet Du Fresnoy, Nicolas: Méthode pour étudier la géographie; où l'on donne une description exacte de l'univers, Tome 9, Paris, N. M. Tilliard https://gallica.bnf.fr/ark:/12148/bpt6k97863330. $\mathrm{r}=$ Istrie? $\mathrm{rk}=21459 ; 2$

1769. - D’Anville, Jean-Baptiste Bourguignon: Géographie ancienne abrégée, Paris, Merlin https://gallica.bnf.fr/ark:/12148/bpt6k5567567x. $\mathrm{r}=$ Istrie? $\mathrm{rk}=21459 ; 2$

1771. - Bellin, Jacques-Nicolas: Description géographique du golfe de Venise et de la Morée, avec des remarques pour la navigation, Paris, Didot https:/gallica.bnf.fr/ark:/12148/bpt6k106709d.r=Istrie?rk=64378;0

1771. - Fortis, Alberto: Saggio d'osservazioni sopra l'isola di Cherso ed Osero, in Venezia, Gaspare Storti http://digital.onb.ac.at/OnbViewer/ viewer.faces?doc=ABO_\%2BZ156785205

1774. - Brion de La Tour, Louis: Atlas, et tables élémentaires de géographie, ancienne et moderne, Paris, J. Barbou https://gallica.bnf.fr/ ark:/12148/bpt6k6532440r.r=Istrie?rk=922751;2

1774. - Fortis, Alberto: Viaggio in Dalmazia dell'abate Alberto Fortis, Venezia, Alvise Milocco https://books.google.hr/ books?id=9WINXtcDAqsC\&hl=hr\&source=gbs_navlinks_s

1777. - Baretti, Giuseppe: Voyage de Londres à Gênes, Tome 2, Passant par l'Angleterre, le Portugal, l'Espagne et la France, Amsterdam, M. M. 
Rey https://gallica.bnf.fr/ark:/12148/bpt6k65338532.r=Istrie?rk=21459;2

1778. - Busching, Anton Friedrich: Nouva geografia, 23 tomo, la continuazione della Marca Trevigiana, il Bassanese, il Feltrino, il Friuli, l'Istria, la Dalmazia, ed il Levante, Venezia, Antonio Zatta https://books. google.hr/books?id=nrH66s_Rd3QC\&hl=\&source=gbs_api\&redir_esc $=y$

1778. - Hacquet, Belsazar: Oryctographia Carniolica, oder Physikalische Erdbeschreibung des Herzogthums Krain, Istrien, I, Leipzig, J. Gottlob \& I. Breitkopf http://reader.digitale-sammlungen.de/de/fs1/object/display/ bsb10226275_00005.html

1781. - Hacquet, Balthasar: Oryctographia Carniolica, oder Physikalische Erdbeschreibung des Herzogthums Krain, Istrien, und zum Theil der benachbarten Länder, II, Leipzig, Breitkopf http://reader.digitalesammlungen.de/de/fs1/object/display/bsb10226276_00005.html

1785. - Morin de La Baume: Lecons de géographie ancienne et moderne, abrégées d'une forme nouvelle, \& sur les observations du capitaine Cook..., Paris, Nyon le jeune https:/gallica.bnf.fr/ark:/12148/ bpt6k97860136.r=Istrie?rk=4077273;2

1788. - Carli, Gian Rinaldo: Delle antichitá italiche, Milano, nell’imperial monistero di S. Ambrogio maggiore https://babel.hathitrust.org/cgi/ pt?id=uiuo.ark:/13960/t9d51zp33;view=lup;seq=1

1792. - Watkins, Thomas: Travels through Switzerland, Italy, Sicily, the Greek Islands to Constantinople, through part of Greece, Ragusa, and the Dalmatian Isles, Vol. I-II, London, T. Cadel https://archive.org/details/ travelsthroughsw01watk/page/n7

1793. - Geographisch-Statistische Beschreibung von See-Österreich und der cisalpinischen Republik. Nebst einer Landcharte worauf die Theilung des venetianischen Staats ..., Leipzig, Linke http://digital.onb.ac.at/ OnbViewer/viewer.faces?doc=ABO_\%2BZ258852803

1794. - Watkins, Thomas: Travels through Switzerland, Italy, Sicily, the Greek islands to Constantinople, through part of Greece, Ragusa, and the Dalmatian isles, vol. 1-2, London, J. Owen https://babel.hathitrust.org/ cgi/pt?id=chi.24755140;view=1up;seq=1 https://babel.hathitrust.org/cgi/ 
pt?id=chi.34336033;view=1up;seq=1

1798. - A Geographical and Statistical Account of the Cisalpine Republic and Maritime Austria, with a Map, Describing the Partition of the Venetian Territory ..., London, G.G. and J. Robinson https://books. google.hr/books/about/A_Geographical_and_Statistical_Account_o. html?id=7AQ8AAAAMAAJ\&redir_esc=y

1798. - /S.n./: Kurzgefaßte geographisch-statistische Beschreibung des k.k. Herzogthums Venedig, und des damit verbundenen Dalmatien und Albanien, Nebst einer Landkarte und dem Plan von Venedig, Wien, Carl Barth https://books.google.hr/ books?id=NvESdSBiYOYC\&hl=hr\&source=gbs_navlinks_s 1802. - Da Mosto, Alvise: Il portolano del mare, Venezia, Gnoato http:// digital.onb.ac.at/OnbViewer/viewer.faces?doc=ABO_\%2BZ15531240X 1802. - Lavallee, Joseph: Voyage pittoresque et historique de l'Istrie et Dalmatie, redige d'apres l'itineraire, ouvrage orne d'estampes, cartes et plans, dessines et leves sur les lieux de L. F. Cassas, Paris, P. Didot Laine http://digi.ub.uni-heidelberg.de/diglit/lavallee1802/0006/image

1805. - A Collection of modern and contemporary voyages and travels: I. Translations from foreign languages, of voyages and travels never before translated, London, Printed for Richard Phillips by Barnard \& Sultzer http://dbooks.bodleian.ox.ac.uk/books/PDFs/590247227.pdf 1805. - Batthyány, Vincenz: Ueber das ungrische Küstenland, Pest, K.A. Hartleben http://digital.onb.ac.at/OnbViewer/viewer. faces?doc $=$ ABO_\%2BZ168015701

1805. - Cassas, Louis F., Joseph Lavallé: Travels in Istria and Dalmatia, drawn up from the itinerary of L.F. Cassas ..., London, for Richard Phillips by J.G. Barnard http://www.dlib.si/details/URN:NBN:SI:docZ4M92NXC http://www.istrianet.org/istria/illustri/non-istrian/cassas/ travels/index.htm

1805. - Von Seenus, Joseph: Beschreibung einer Reise nach Istrien und Dalmatien vorzüglich in botanischer Hnsicht, Nürnberg, Monath und Kußler http://reader.digitale-sammlungen.de/de/fs1/object/display/ 
bsb10477219_0000w1.html

1805. - Wiedemann, Joseph G.: Streifzüge an Istriens Küsten,

Wien, A. Doll http://digital.onb.ac.at/OnbViewer/viewer.

faces?doc=ABO_\%2BZ161291905

1808. - Boucher de La Richarderie, Gilles: Bibliothèque universelle des voyages, ou Notice complète et raisonnée de tous les voyages anciens et modernes ..., T. 2, Paris, chez Treuttel et Würtz, ancien hôtel de Lauraguais https://gallica.bnf.fr/ark:/12148/bpt6k6546295j. $\mathrm{r}=$ Istrie? $\mathrm{rk}=21459 ; 2$

1808. - De Rossel, Elisabeth-Paul-Edouard: Voyage de Dentrecasteaux envoyé à la recherche de La Pérouse, Tome 1, Paris, Impr. Impériale https://gallica.bnf.fr/ark:/12148/bpt6k9782601t.r=Istrie?rk=21459;2

1808. - Hoff, Heinrich Georg: Historisch-statistisch-topographisches Gemälde vom Herzogthume Krain, und demselben einverleibten Istrien, ein Beytrag zur Völker- und Länderkunde, Laibach, H. W. Korn https:// babel.hathitrust.org/cgi/pt?id=hvd.hxj9eq;view=lup;seq=1

1809. - Delamarche, Charles-François: Description géographique et historique des peuples les plus renommés de l'Europe ancienne et des lieux les plus remarquables..., Un atlas composé de 18 cartes vient à l'appui..., Paris, Delamarche https://gallica.bnf.fr/ark:/12148/ bpt6k432433g.r=Istrie?rk=1802584;0

1810. - Widemann, Joseph Georg: Streifzüge an Istriens

Küsten, Wien, Doll http://digital.onb.ac.at/OnbViewer/viewer. faces?doc=ABO_\%2BZ223466809

1812. - Allason, Thomas: Picturesque views of the antiquities of Pola, in Istria, London, J. Murray https://www.openstarts.units.it/ bitstream/10077/13142/1/DZ1ARU001471.pdf

1812. - Von Woltersdorf: Die Illyrischen Provinzen und ihre Einwohner, Wien, Camesina http://reader.digitale-sammlungen.de/de/fs1/object/ display/bsb10721390_00005.html

1815. - A tour through some parts of Istria, Carniola, Styria, Austria, the Tyrol, Italy and Sicily ..., by a young English merchant, London, Gale and 
Fenner http://dbooks.bodleian.ox.ac.uk/books/PDFs/590528656.pdf 1816. - Vallardi, Pietro G.: Itinerario italiano, Descrizione dei viaggi per le strade più frequentate alle principali città d'Italia, coll' indicazione delle distanze in poste ..., Milano, Pietro e Giuseppe Vallardi https://babel. hathitrust.org/cgi/pt?id=gri.ark:/13960/t4rj8s22n;view=lup;seq=1 1817. - Itinerario italiano, Descrizione di viaggi 52 per le strade più frequentate alle principali città d'Italia, coll'indicazione delle distanze in poste, in miglia, in ore e minute ..., Firenze, Niccolò Pagni https://babel. hathitrust.org/cgi/pt?id=nnc1.1002585723; view=1up;seq=1 1817. - Pannelier, Jean Amable: Nouvel abrégé des géographies de Nicole de La Croix, Crozat et Lenglet-Dufresnoy, par demandes et par réponses ..., Paris, A. Delalain https://gallica.bnf.fr/ark:/12148/bpt6k65339698. $\mathrm{r}=$ Istrie? $\mathrm{rk}=2618038 ; 4$

1819. - Allason, Thomas: Picturesque views of the antiquities of Pola in Istria, the plates engraved by W. B. Cooke ... and Cosmo Armstrong, London, John Murray https://arachne.uni-koeln.de/arachne/index. php?view[layout]=buchseite_item\&search[constraints][buchseite][buch. origFile $]=$ BOOK-ZID192461. $x m$ \& $\&$ view $[$ page $]=0$

1821. - Bassi, Domenico Giovanni: Costiere Del Mare Adriatico Ovvero Descrizione Di Tutti I Porti, Rade, Baje, Isole Ec., Venezia, Francesco Andreola http://digital.onb.ac.at/OnbViewer/viewer. faces?doc $=$ ABO_\%2BZ16870600X

1821. - Memorie politico-economiche della città e territorio di Trieste, della penisola d'Istria, della Dalmazia fu Veneta, di Ragusi e dell'Albania ..., Venezia, Alvisopoli http://reader.digitale-sammlungen.de/de/fs1/ object/display/bsb10010457_00001.html

1821. - Shoberl, Frederic: Illyria and Dalmatia, containing a description of the manners, customs, habits, dress, and other peculiarities characteristic of their inhabitants ..., London, F. Ackerman https://archive.org/details/ ahy8052.0001.001.umich.edu

1822. - Medesimo, Lui: Relazione di un viaggio a Costantinopoli di Giambattista Casti nel 1788..., Milano, Tip. Batelli e Fanfani https:// 
books.google.hr/books?id=RR3I8oYdWMAC\&hl=hr\&source=gbs navlinks_s

1822. - Stancovich, Pietro: Dello anfiteatro di Pola, dei gradi marmorei del medesimo, nuovi scavi e scoperte e di alcune epigrafi e figuline inedite dell'Istria, con VIII tavole, Venezia, Giuseppe Picotti https://babel. hathitrust.org/cgi/pt?id=gri.ark:/13960/t5r83693v;view=1up;seq=1

1822. - Von Liechtenstern, Joseph Marx Freiherr: Reisen durch das österreichische Illyrien, Dalmatien und Albanien im Jahre 1822., Th. 1, Meissen, Goedsche http://digital.onb.ac.at/OnbViewer/viewer. faces?doc=ABO_\%2BZ181189503

1823. - Von Jenny, R. E.: Handbuch für Reisende in dem österreichischen Kaiserstaate, mit mehreren Hauptrouten der angränzenden Länder, 1-2, Wien, A. Doll https://babel.hathitrust.org/cgi/ pt?id=wu.89072317167;view=1up;seq=1

1824. - Michelot, Henry: Portulan de la Mer Méditerranée, ou guide des pilotes côtiers (... la description des côtes du Levant et des isles de l'Archipel, et d'un extrait des routes et distances d'un port à un autre en milles ...), Marseille, Jean Mossy https://gallica.bnf.fr/ark:/12148/ bpt6k9677831p.r=Istrie?rk=5751101;2

1828. - Stancovich, Pietro: Biografia degli uomini distinti dell'Istria, 1-2, Trieste, Marenich http://reader.digitale-sammlungen.de/de/fs1/object/ display/bsb10071009_00001.html http://reader.digitale-sammlungen.de/ de/fs1/object/display/bsb10071010_00001.html

1830. - Davy, Humphry: Consolations in Travel, Or, The Last Days of a Philosopher, London, John Murray https://books.google.hr/ books?id=KiANAAAAYAAJ\&hl=hr\&source=gbs_navlinks_s

1830. - Frankland, Charles Colville: Travels to and from Constantinople in the years 1827 and $1828, \ldots$ a journey from Vienna ... to Constantinople ..., Malta, Italy, Istria, Carniolia, and Styria, London, H. Colburn http://dbooks.bodleian.ox.ac.uk/books/PDFs/303978423.pdf 1830. - Lichtenthal, Peter: Manuale bibliografico del viaggiatore in Italia concernente località, storia, arti, scienze ed 
antiquaria, Milano, Antonio Fontana https://books.google.hr/ books?id=N1BSz9VE1P4C\&hl=hr\&source=gbs_navlinks_s 1830. - Marieni, Giacomo: Portolano Del Mare Adriatico Compilato Sotto La Direzione Dell'Istituto Geografico Militare, Milano, Dall' Imp. Regia Stamp. http://digital.onb.ac.at/OnbViewer/viewer. faces?doc=ABO_\%2BZ166432904

1831. - Groß-Hoffinger, Anton Johann: Handbuch für Reisende durch das Erz-Herzogthum Österreich, Steiermark, Salzburg, Krain, Kärnten, Tirol, Illirien, Dalmatien und das lombardisch-venetianische Königreich, München, J. Linbaner http://reader.digitale-sammlungen.de/de/fs1/object/ display/bsb10466925_00005.html

1833. - Grubas, G. B.V.M.: Nuovo costiere del mare Adriatico, Trieste, Orlandini Figlio http://digital.onb.ac.at/OnbViewer/viewer. faces?doc=ABO_\%2BZ166666800

1834. - Lichtenthal, Pietro: Manuale bibliografico del viaggiatore in Italia concernente località, storia, arti, scienze, antiquaria e commercio preceduto ..., Milano, per Luigi di Giacomo Pirola https://www.e-rara.ch/ zut/content/titleinfo/7407541?query=Istria

1834. - Nuovissima guida dei viaggiatori in Italia, Milano, Artaria Epimaco e Pasquale https://babel.hathitrust.org/cgi/ pt?id=nnc1.1002587181;view=1up;seq=1

1836. - Spencer, Edmund: Sketches of Germany and the Germans, with a glance at Poland, Hungary, \& Switzerland, vol. 1-2, London, Whittaker https://babel.hathitrust.org/cgi/ pt?id=nyp.33433066655345;view=1up;seq=1 https://babel.hathitrust.org/ cgi/pt?id=nyp.33433066655337;view=1up;seq=1

1837. - Koch, Wilhelm Daniel Joseph: Synopis der deutschen und schweizer Flora, enthaltend die genauer bekannten Pflanzen, welche in Deutschland, der Schweiz, in Preussen und Istrien wild wachsen ..., Frankfurt a. M., Wilmans http://reader.digitale-sammlungen.de/de/fs1/ object/display/bsb10304139_00005.html

1838. - Sartorio, Giovanni Guglielmo: Viaggio di sua maesta Federico 
Augusto, re di Sassonia alla Dalmazia, Zara, Tipografia fratelli Battara https://digitalna.nsk.hr/pb/?object=view\&id=12447

1840. - De Casotti, Marco (Marko Kažotić): Le coste e isole della Istria e della Dalmazia, Zara, Tipografia Battara http://reader.digitalesammlungen.de/de/fs1/object/display/bsb10009220_00001.html 1840. - Löwenthal, Jakob: Der Istrianer Kreis oder die Halbinsel Istrien und die Inseln des Quarnero, mit einer topographischen Karte ..., und einer Uebersicht der ökonomischen Pflanzen in Istrien von Dr. B. Biasoletto, Wien, H. H. Müller http://reader.digitale-sammlungen.de/de/ fs1/object/display/bsb10804575_00024.html

1840. - Ternaux-Compans, Henri: Archives des voyages, Collection d'anciennes relations inédites ou très-rares de lettres mémoires itinéraires ... et aux voyages et d'anecdotes relatives aux voyageurs...., T. 2, Paris, A. Bertrand, 1840-1841. https://gallica.bnf.fr/ark:/12148/bpt6k65744978. $\mathrm{r}=$ Istrie? $\mathrm{rk}=21459 ; 2$

1840. - Zucoli, Luigi: Nuovissima Guida Dei Viaggiatori In Italia Coll’Aggiunta Dei Viaggi Nella Dalmazia, Nell’Istria E Nella Grecia Per La Prima Volta Pubblicati ... Corredata Della Carta D'Italia ..., Milano, L. Zucoli http://digital.onb.ac.at/OnbViewer/viewer. faces?doc $=$ ABO_\%2BZ221139303 1841. - Turnbull, Peter Evan: Reise durch die Oesterreichischen Staaten, Leipzig, Weber http://digital.onb.ac.at/OnbViewer/viewer. faces?doc $=$ ABO_\%2BZ176512407

1842. - Biasoletto, Bartolomeo: Reise Sr. Majestät des Königs Friedrich August von Sachsen durch Istrien, Dalmatien und Montenegro im Frühjahr 1838, Dresden, Gottschalk http://digital.slub-dresden.de/en/ workview/dlf/102787/1/

1842. - Biasoletto, Bartolomeo: Relazione del viaggio fatto nella primavera dell'anno 1838 dalla maesta del re Federico Augusto nell'Istria, Dalmazia e Montenegro, Trieste, H. F. Favarger http://reader.digitale-sammlungen. de/de/fs1/object/display/bsb10483444_00013.html

1843. - Versuch einer Geschichte und Beschreibung der Stadt Pola in 
Istrien..., Triest, Favarger http://digital.onb.ac.at/OnbViewer/viewer. faces?doc=ABO_\%2BZ170500705

1844. - Solitro, V.: Documenti storici sull'Istria e la Dalmazia, Venezia, Gattei http://reader.digitale-sammlungen.de/de/fs1/object/display/ bsb10136585_00001.html

1845. - Kandler, Pietro: Cenni al forestiero che visita Parenzo, Trieste, Papsch http://digital.onb.ac.at/OnbViewer/viewer. faces?doc=ABO_\%2BZ205942301 1845. - Kandler, Pietro: Cenni Al Forestiero che visita Pola, Trieste, Papsch http://digital.onb.ac.at/OnbViewer/viewer. faces?doc=ABO_\%2BZ176674900

1845. - Stieglitz, Heinrich Wilhelm August: Istrien und Dalmatien, Briefe und Erinnerungen, Stuttgart, Tuebingen, J.G. Cotta’schen Buch. http:// reader.digitale-sammlungen.de/resolve/display/bsb11249623.html 1848. - Morlot, Adolphe: Über die geologischen Verhältnisse von Istrien ..., Wien, Baumüller und Seidel http://reader.digitale-sammlungen.de/de/ fs1/object/display/bsb10226245_00005.html

1848. - Wilkinson, John Gardner: Dalmatia and Montenegro ..., The History of Dalmatia and Rugusa, vol. 1, 2., London, John Murray http:// dbooks.bodleian.ox.ac.uk/books/PDFs/N11982281.pdf http://dbooks. bodleian.ox.ac.uk/books/PDFs/N11982282.pdf

1849. - Chmel, Joseph: Urkunden zur Geschichte von Österreich, Steiermark, Kärnten, Krain, Görz, Triest, Istrien, Tirol ..., Wien, K.K. Hof- und Staatsdr. http://reader.digitale-sammlungen.de/de/fs1/object/ display/bsb10798115_00005.html

1851. - Kohl, Johann Georg: Reise nach Istrien, Dalmatien und Montenegro, Dresden, Arnoldische Buchhandlung http://reader.digitalesammlungen.de/de/fs1/object/display/bsb10467182_00005.html 1852. - Champagnac, Jean-Baptiste-Joseph: Ernest et Fortunat ou Les jeunes voyageurs en Italie ..., Paris, Lehuby https://gallica.bnf.fr/ ark:/12148/bpt6k6566989s.r=Istrie?rk=1824043;2 
1852. - Triest und seine Umgebungen, ein Wegweiser für Fremde und Einheimische, Triest, Verlag der artist. liter. Abtheil. des österr. Lloyd https://babel.hathitrust.org/cgi/ pt?id=inu.32000002529891; view=1up;seq=1

1854. - Marmier, Xavier: Lettres sur l'Adriatique et le Monténégro, T. 1., Paris, Arthus Bertrand https:/gallica.bnf.fr/ark:/12148/bpt6k106227s. $\mathrm{r}=$ Istrie? $\mathrm{rk}=21459 ; 2$

1855. - Kandler, Pietro: Indicazioni per riconoscere le cose storiche del Litorale, Einen Theil dieses Werkes bilden die "Inscrizioni Romane dell' Istria”, Trieste, Lloyd http://reader.digitale-sammlungen.de/de/fs1/object/ display/bsb10000228_00001.html

1856. - Koch, Wilhelm Daniel Joseph: Taschenbuch der deutschen und Schweizer Flora, enthaltend die genauer bekannten Pflanzen, welche in Deutschland, der Schweiz, in Preussen und Istrien ..., Leipzig, Gebhardt und Reisland http://reader.digitale-sammlungen.de/de/fs1/object/display/ bsb10302064_00005.html

1857. - Rieger, G.: Panorama della Costa e delle Isole di Dalmazia nei viaggi dei piroscafi del Lloyd Austriaco, Trieste, Linassi https://www. youtube.com/watch?v=GWZ5fjLpn_8

1858. - /Cantù, Cesare/: Escursione pel litorale dell'Istria, /Trieste/, /s.n./ https://www.openstarts.units.it/bitstream/10077/20536/1/AS_ASA003924. pdf

1859. - Von Baude, Jean Jacques: Oestreich's adriatische Küste und Seemacht, Calamota-Triest-Pola, Hamburg, Hoffmann u. Campe http:// digital.onb.ac.at/OnbViewer/viewer.faces?doc=ABO_\%2BZ25722870X 1861. - Grube, Eduard: Ein Ausflug nach Triest und dem Quarnero, Grube, Berlin, Nicolaische verlag https://archive.org/details/ einausflugnachtr00grub 1861. - Neale, J.M.: Notes, Ecclesiological and Picturesque, on Dalmatia, Croatia, Istria, Styria, with a Visit to Montenegro, London, J. T. Hayes http://dbooks.bodleian.ox.ac.uk/books/PDFs/600023388.pdf 1863. - De Budahegy, Pauer C. - Giovanni, Carlo: Sulla necessita 
d'un aumento dell'illuminazione marittima lungo le coste del mare adriatico ..., Fiume, Huber http://digital.onb.ac.at/OnbViewer/viewer. faces?doc=ABO_\%2BZ219445907

1863. - Durand, Hippolyte: Le Danube Allemand et l'Allemagne du Sud voyage dans la Foret-Noire ... la Bohème, la Hongrie, l'Istrie, la Vénetie et le Tyrol..., Tours, Mame et cie. https://babel.hathitrust.org/cgi/pt?id=hvd. hn2x6x;view=1up;seq=1

1863. - Köhler, Johann: Istrien, historische, geographische und statistische Darstellung der Istrischen Halbinsel nebst den Quarnerischen Inseln, Triest, Österr. Lloyd http://reader.digitale-sammlungen.de/de/fs1/object/ display/bsb10010092_00005.html

1863. - Von Goracuchi, J. Alexander: Die Adria und ihre Küsten ...nebst einer Erörterung über das Seewasser und dessen heilbringende Wirkung, Triest, Buchdruck. d. österr. Lloyd http://reader.digitale-sammlungen.de/ de/fs1/object/display/bsb10405955_00005.html

1864. - Beaufort Smythe Strangford, Emily Anne: The Eastern Shores of the Adriatic in 1863, With a Visit to Montenegro, London, R. Bentley https://books.google.hr/books?id=kaUKAAAAYAAJ

1864. - Costantini, Carlo: Guida pratica per la navigazione del mare Adriatico e delle isole Jonie da Corfu a Cerico ..., Trieste, C. Coen http:// digital.onb.ac.at/OnbViewer/viewer.faces?doc=ABO_\%2BZ226074207 1865. - /S.n./: Venetië, De Aarde en haar Volken, /Denmark/ http://www.gutenberg.org/ebooks/14112.epub.images?session $\mathrm{id}=3403 \mathrm{~d} 7 \mathrm{c} 22 \mathrm{aee} 7 \mathrm{c0fdb} 8 \mathrm{f} 6 \mathrm{a} 0 \mathrm{c} 5 \mathrm{fe} 2609 \mathrm{f} 4 \mathrm{fab} 19 \mathrm{~b} 0$

1866. - Von Babo, A.: Bericht über die im Auftrage des K. K. ... Bereisung der Wimbau treibenden Kronländer Oesterreichs: Kroatien, Dalmatien, Istrien ..., Wien, C. Gerold's Söhne http://reader.digitale-sammlungen. de/de/fs1/object/display/bsb10293867_00005.html

1867. - Gareis, Anton: Pola und seine nächste Umgebung, Triest, F. H. Schimpff http://digital.onb.ac.at/OnbViewer/viewer. faces?doc $=$ ABO_\%2BZ153167407

1867.- Kandler, Pietro: Discorso sull'Istro Adriaco, Trieste, 
Lloyd Austriaco http://digital.onb.ac.at/OnbViewer/viewer. faces?doc $=$ ABO_\%2BZ218096000

1872. - Baedeker, Karl: Südbayern, Tirol und Salzburg, Steiermark, Kärnthen, Krain und Istrien, Handbuch für Reisende, Coblenz, Baedeker http://reader.digitale-sammlungen.de/de/fs1/object/display/ bsb11254863_00005.html

1872. - Driou, Alfred: Les Apennins et la mer Adriatique, Limoges, Barbou frères https://gallica.bnf.fr/ark:/12148/bpt6k1069180. $\mathrm{r}=$ Istrie? $\mathrm{rk}=3605168 ; 0$

1873. - Von Oesterreicher, Tobias: Die Oesterreichische Küstenaufnahme im Adriatischen Meere, Triest, Appolonio und Caprin http://digital.onb. ac.at/OnbViewer/viewer.faces?doc=ABO_\%2BZ224354006

1874. - De Perrochel, Fernand: Une semaine en Istrie, Le Mans, E. Monnoyer https://gallica.bnf.fr/ark:/12148/bpt6k107070h. $\mathrm{r}=$ Istrie? $\mathrm{rk}=21459 ; 2$

1874. - Maiorescu, Ioan: Itinerar in Istria, și Vocabular Istriano-Român, Jassi, Tip. Litografia H. Goldner https://books.google.hr/books/about/ Itinerar_in_Istria.html?id=XyMdnQEACAAJ\&redir_esc=y 1875. - Du Prel, Carl: Unter Tannen und Pinien, Wanderungen in den Alpen, Italien, Dalmatien und Montenegro, Berlin, Denicke http://reader. digitale-sammlungen.de/de/fs1/object/display/bsb11316253_00005.html 1875. - Leger, Louis: Études slaves, voyages et littératures..., Paris, E. Leroux https://gallica.bnf.fr/ark:/12148/bpt6k56753852. $\mathrm{r}=$ Istrie? $\mathrm{rk}=5665264 ; 0$

1875. - R.H.R.: Rambles in Istria, Dalmatia ..., London, Hurst and Blackett http://dbooks.bodleian.ox.ac.uk/books/PDFs/600020877.pdf 1875. - Yriarte, Charles: Trieste e l'Istria, Milano, Fratelli Treves https:// babel.hathitrust.org/cgi/pt?id=hvd.32044082265802;view=lup;seq=1 http://www.istrianet.org/istria/illustri/non-istrian/yriarte/works/triesteistria/index.htm

1877. - Benussi, Bernardo, Manuale di geografia dell’Istria, 
Trieste, Caprin http://digital.onb.ac.at/OnbViewer/viewer. faces?doc=ABO_\%2BZ219241901

1877. - Burton, Richard Francis: Note sopra i Castelliere o rovine preistoriche nella penisula istriana, Capodistria, Stab. Tip. B. Appolonio http://digital.onb.ac.at/OnbViewer/viewer. faces?doc=ABO_\%2BZ254294708

1877. - Garimberti, Cesare: Diario storico del viaggio di ... Francesco Giuseppe I. imperatore d' Austria ... a Trieste, Gorizia, Venezia, in Istria, in Dalmazia ed a Fiume ..., Zara, Vitaliani e Janković http://digital.onb. ac.at/OnbViewer/viewer.faces?doc=ABO_\%2BZ254112904

1878. - Yriarte, Charles: Les bords de l'Adriatique et le Monténégro, Venise, l' Istrie, le Quarnero, la Dalmatie, le Monténégro et la rive italienne, Paris, Hachette https://gallica.bnf.fr/ark:/12148/bpt6k106870q. $\mathrm{r}=$ Istrie? $\mathrm{rk}=193134 ; 0$

1881. - Freeman, Edward A.: Sketches from the Subject and Neighbor Lands of Venice, London, Macmillan and Co. https://babel.hathitrust. org/cgi/pt?id=mdp.39015063901923;view=1up;seq=21

1882. - De Nolhac, Stanislas: La Dalmatie, les îles Ioniennes, Athènes et le mont Athos, Paris, E. Plon https://gallica.bnf.fr/ark:/12148/bpt6k2029827. $\mathrm{r}=$ Istrie? $\mathrm{rk}=85837 ; 2$

1883. - Tissot, Victor: La Hongrie de l'Adriatique au Danube, impressions de voyage, ouvrage illustré de 10 héliogravures d'après Valerio ..., Paris, E. Plon et Cie https://gallica.bnf.fr/ark:/12148/bpt6k1025220q. $\mathrm{r}=$ Istrie? $\mathrm{rk}=3369115 ; 2$

1883. - Von Schweiger-Lerchenfeld, Amand Freiherr: Die Adria, Land und Seefahrten im Bereiche des Adriatischen Meeres ..., Wien, Pest, Leipzig, A. Harteleben's Verlag http://digital.bib-bvb=0\&dvs=1539002008 533 $749 \&$ pid $=1493354 \&$ locale $=$ en\&usePid $1=$ true\&usePid $2=$ true 1883. - Von Schweiger-Lerchenfeld, Amand Freiherr: L'Adriatico e il suo litorale, con numerose incisioni originali ..., Milano, Antica casa editrice Francesco Vallardi https://www.openstarts.units.it/ bitstream/10077/20524/1/AS_ASA003867.pdf 
1883. - Yriarte, Charles: Le rive dell'Adriatico e il Montenegro, illustrato da 261 incisioni e 2 carte, Venezia, Chioggia, Trieste, l'Istria, il Quarnero e le sue isole, la Dalmazia, il Montenegro ..., Milano, Fratelli Treves https://archive.org/details/lerivedelladriat00yria 1887. - Jackson, Thomas Graham: Dalmatia, the Quarnero and Istria, with Cettigne in Montenegro and the island of Grado, Oxford, Clarendon http://dbooks.bodleian.ox.ac.uk/books/PDFs/N11523027.pdf

1888. - Bauron, Pierre: Les rives illyriennes, Istrie, Dalmatie, Monténégro, ouvrage illustré d'une carte \& de 34 gravures inédites, Paris, Retaux-Bray https://archive.org/details/lesrivesillyrien00baur

1888. - Heer, Jakob Cristoph: Ferien an der Adria, Bilder aus SüdÖsterreich, Frauenfeld und Leipzig, Verlag von Huber \& Co. http://www.gutenberg.org/ebooks/50888.epub.images?session_ id=b537edd1f9d958d11695a6833a4023e0daae3ed7

1888. - Noë, Heinrich: Wanderungen und Bilder, in und aus dem österreichischen Küstenlande, Krain, Istrien ..., Glogau, Carl Flemming http://digital.tessmann.it/tessmannDigital/Medium/Seite/21066/3

1892. - Playfair, R. Lambert: Handbook to the Mediterranean; its cities, coasts and islands, For the use of general travellers and yachtsmen, London, J. Murray https://babel.hathitrust.org/cgi/ pt?id=mdp.39015068284887;view=lup;seq=1

1894. - Bernard, Marius: Autour de la Méditerranée.... l'Autriche et la Grèce, De Venise à Salonique, Paris, H. Laurens, 1894-1902. https:// gallica.bnf.fr/ark:/12148/bpt6k6547754n.r=Istrie?rk=21459;2

1895. - Gobbi Belcredi, Giacomo: Nell Istria, con quindici illustrazioni dal vero, Roma, Stab. tip. ital. https://digitalna.nsk.hr/ $\mathrm{pb} /$ ?object $=$ info\&id $=11355$

1895. - Lesker, Bernhard: Eine Fahrt an die Adria, Stuttgart, Süddeutsche Verlagsbuchhandlung (D. Ochs) https://archive.org/details/ afn6245.0001.001.umich.edu

1896. - Maury, Édouard: Aux portes de l'Orient, la lagune de Venise, Istrie et Dalmatie, Herzégovine et Bosnie, Montenegro ..., 
Paris, Fischbacher https://gallica.bnf.fr/ark:/12148/bpt6k106124b. $\mathrm{r}=$ Istrie? $\mathrm{rk}=686698 ; 4$

1898. - Miller, William: Travels and Politics in the Near East, Chapter 1., The Threshold of the Near East, Istria and Dalmatia, New York, Frederick A. Stokes Co. https://archive.org/details/travelspolitic00mill 1899. - Illustrierter Führer durch Dalmatien (Abbazia - Lussin) längs der Küste von Albanien bis Korfu und nach den Ionischen Inseln, Wien, Hartleben Verlag https://cdk.lib.cas.cz/client/ index.vm?q $=$ Istrie \&page $=$ doc\&start $=0 \&$ rows $=\&$ fedora . model=monograph\#pid=uuid:a06d0bd0-8fc0-11e2-8e050030487be43a;hist=2

1899. - Marcotti, Giuseppe: L’ Adriatico orientale, da Venezia a Corfù, guida illustrata, Firenze, R. Bemporad \& Figlio https://www.openstarts. units.it/bitstream/10077/13309/8/BG_BGA053997.pdf 1899. - Petermann, Reinhard E: Fuehrer durch Dalmatien ..., Wien, Alfred Hoelder https://archive.org/details/fuhrerdurchdalma00peteiala 1900. - Bonifacio, Giacomo: Approdo di Pio 7. in Istria nel viaggio da Venezia a Pesaro, Capodistria, Tipografia Cobol \& Priora https://www. dlib.si/stream/URN:NBN:SI:DOC-5OP9J9IT/0ce07154-9f1b-49ac-b4fa553d52f1e02c/PDF

1901. - Il Lloyd austriaco ed i paesi dei suoi itinerari, manuale ufficiale di viaggio: Istria, Dalmazia, Erzegovina e Bosnia, Soc. Lloyd austriaco, Vienna, Rohrer https://www.openstarts.units.it/bitstream/10077/16035/1/ AS_ASA002433.pdf

1902. - Baumberger, Georg: Blaues Meer und schwarze Berge, Volks und Landschaftbilder aus Krain, Istrien, Dalmatien, Montenegro, New York, Benziger https://babel.hathitrust.org/cgi/ pt?id=umn.319510023217564;view=lup;seq=7

1903. - Silvestri, Emilio: L'Istria, Opera riccamente illustrata da incisioni e Fotozincotipie, Vicenza, G. Rumor https://archive.org/details/ SilvestriIstria

1903. - Stradner, Joseph: Novi schizzi dall’Adria II., Istria, Trieste, 
F. H. Schimpff http://asa.archiviostudiadriatici.it/islandora/object/ libria:205542/datastream/PDF/content/libria_205542.pdf 1905. - Brown, Horatio F.: In and Around Venice, New York, C. Scribner's Sons https://babel.hathitrust.org/cgi/ pt?id=hvd.32044082268426;view=lup;seq=17 1908. - Jackson, Frederick Hamilton: The shores of the Adriatic, the Austrian side, the Küstenlande, Istria, and Dalmatia, London, John Murray https://babel.hathitrust.org/cgi/ pt?id=nyp.33433066663737;view=1up;seq=22

1913. - Baker, James: Austria: Her people and their Homelands, Ch. XVI (Triest and Istria) and XVII (Down the Istrian Coast to Dalmatia, to Sebenico), London, J. Lane https://archive.org/details/ austriaherpeople00bake

1915. - Ryan, Nellie: My Years at the Austrian Court, London, John Lane https://archive.org/details/myyearsataustria00ryan

1916. - Brunialti, Attilio: Trento e Trieste, Dal Brennero alle rive dell'Adriatico ..., Parte 5. - L'Istria di San Marco, Parte 6. - Fiume e il Quarnaro, Torino, Un. Tip.-Editrice Torinese https://archive.org/details/ BrunialtiTrentoTriesteIstriaFiume 1918. - Cvijić, Jovan: La Péninsule balkanique, géographie humaine..., Paris, A. Colin https://gallica.bnf.fr/ark:/12148/bpt6k5530552g. $\mathrm{r}=$ Istrie? $\mathrm{rk}=708158 ; 0$ 1920. - The Austrian Littoral, Historical Section of the Foreign Office, London, H.M. Stat. Office https://babel.hathitrust.org/cgi/ pt?id=njp.32101045249412;view=1up;seq=1

1923. - Jackson, Thomas Graham: Memories of Travel, Cambridge, The University Press http://www.istrianet.org/istria/illustri/non-istrian/ jackson_tg/works/memories_of_travel/index.htm 


\section{REFERENCES}

Bertoša, S. - A. Giudici (2013): Europski putnici u Istri od XV. do XIX. stoljeća, Tabula, 11, s. 7-35 https://hrcak.srce.hr/index. php?show=clanak\&id_clanak_jezik=172711

Botton, A. (2005): Umijeće putovanja, Zagreb, SysPrint

Carmichael, C. (1996): Etnički stereotipi u ranim europskim etnografijama: proučavanje/ primjer Habsburškoga Jadrana od oko 1770. do 1815., Narodna umjetnost, 33 (2), s. 197-208 https://hrcak.srce.hr/index. php?show=clanak\&id_clanak_jezik=69755

Despot, M. (1955): Strana svjedočanstva o slavenstvu Istre od 16.-18. st., Riječka revija, 4(2), 84-87

Despot, M.: (1953): Strani putopisci o Istri, Jugoslavenski mornar, 1 (s. 24), 2 (s. 48), 3 (s. 72), 4 (s. 96)

Duda, D. (2012): Kultura putovanja, uvod u književnu iterologiju, Zagreb, Naklada Ljevak

Kozličić, M. (2012): Adriatic sea routes from the antiquity to the earl modern age, Histria Antiqua, 21, p. 13-20 https://www.pilar.hr/wp-content/ images/stories/dokumenti/histria_antiqua/21/hi21_01kozlicic.pdf

Kužić, K. (2013): Hrvatska obala u putopisima njemačkih hodočasnika 14.17. st., Split, Književni krug

Levental, Z. (1986): Britanski putnici u našim krajevima, Dečje novine, Gornji Milanovac

Matijašić R. (1998): Putopisi i starinoznanstvo 17. i 18. st. na našoj obali, Nova Istra, 3(10), s. 122-132

Miličić, I. (2010): Teoretičari, hodočasnici, činovnici: tri vrste renesansnih putopisnih tekstova, Povijesni prilozi, 38 (38), s. 43-69 https://hrcak.srce. $\mathrm{hr} / 56766$

Nikolić Đerić, T. (2012): Istra u putopisu Charlesa Yriarteaa, analiza putopisa kao književnog teksta i teksta kulture, Etnološka tribina, 42 (35), s. 137-150 https://hrcak.srce.hr/94121 
Pavić, M. (2007): Prilog poznavanju hodočasničkih putovanja od Venecije do Svete zemlje u XVI. stoljeću, Croatica Christiana periodica, 31 (59), s. 33 47 https://hrcak.srce.hr/index.php?show=clanak\&id_clanak_jezik=31787 Pavić, M. (2016): Perceptions of the Eastern Adriatic in the travel literature of the Early Modern period, Tabula, 14, 103-117 https://hrcak.srce.hr/ file/261399

Pederin, I. (1991): Jadranska Hrvatska u austrijskim i njemačkim putopisima, Zagreb, Nakladni zavod MH

Pederin, I. (2001): Barokni putopisi, putopis misionara, vojnika i političkih putnika, Crkva u svijetu, 36 (3), s. 331-353 https://hrcak.srce.hr/index. php?show=clanak\&id_clanak_jezik=61341

Pederin, I. (2002): Vjerska pozadina putopisa u doba otkrića, Crkva u svijetu, 37 (4), s. 448-473 https://hrcak.srce.hr/index.php?show=clanak\&id_ clanak_jezik=61205

Pederin, I. (2009): Povijesna poetika putopisa, Omiš, Tiskara "Franjo Kluz" 
